\title{
Comparative cardiovascular safety of GLP-1 receptor agonists versus other glucose-lowering agents in real-world patients with type 2 diabetes: a nationwide population-based cohort study
}

Chun-Ting Yang

National Cheng Kung University College of Medicine https://orcid.org/0000-0002-5747-6806

Chen-Yi Yang

National Cheng Kung University College of Medicine

Huang-Tz Ou ( $\square$ huangtz@mail.ncku.edu.tw )

https://orcid.org/0000-0002-5475-7848

Shihchen Kuo

University of Michigan Medical School

Original investigation

Keywords: GLP-1 receptor agonist, cardiovascular safety, DPP-4 inhibitor, sulfonylurea, insulin

Posted Date: February 27th, 2020

DOl: https://doi.org/10.21203/rs.2.24736/v1

License: (1) (1) This work is licensed under a Creative Commons Attribution 4.0 International License.

Read Full License

Version of Record: A version of this preprint was published at Cardiovascular Diabetology on June 13th, 2020. See the published version at https://doi.org/10.1186/s12933-020-01053-0. 


\section{Abstract}

Background : Current evidence about the cardiovascular safety of glucagon-like peptide-1 receptor agonist (GLP-1 ra) possesses limited generalizability to real-world patients with type 2 diabetes (T2D) in usual practice. This study aimed to investigate the comparative cardiovascular safety of GLP-1 ra in headto-head comparisons with dipeptidyl peptidase-4 inhibitor (DPP-4i), sulfonylurea (SU), and insulin in a real-world population with T2D.

Methods : Adults with newly-diagnosed T2D were identified from Taiwan's National Health Insurance Research Database in 2003-2014. A prevalent new-user cohort design was adopted to include a broad representation of real-world T2D patients being treated with GLP-1 ra. The between-group comparability of baseline patient characteristics was achieved by matching on (1) initiation time of study drugs, (2) prior exposure to glucose-lowering agents, and (3) diabetes severity and complications, comorbidities, and concomitant cardiovascular medications using propensity scores. The primary outcome was a composite of cardiovascular disease (CVD) events and assessed up to the end of 2015. Cox modeling was employed to assess the association between study drugs and outcomes.

Results : A total of 3,195 GLP-1 ra stable users was identified in 2011-2014. 1,893, 1,829, and 1,367 GLP1ra stable users were 1:1 matched to DPP-4i, SU and insulin users, respectively. Compared to DPP-4i, SU and insulin, the use of GLP-1ra was associated with a lower risk of composite CVD events (hazard ratio [95\% confidence interval]: 0.73 [0.57-0.96], 0.76 [0.57-1.00], and 0.81 [0.62-1.07], respectively). Subgroup analyses revealed that GLP-1ra versus DPP-4i yielded a greater cardiovascular benefit in those without established CVD versus those with established CVD.

Conclusions: This head-to-head comparison study extends the supporting evidence for the cardiovascular safety of GLP-1 ra to a broad spectrum of real-world T2D patients using GLP-1ra.

\section{Background}

Although early initiation of glucagon-like peptide-1 receptor agonist (GLP-1 ra) is recommended for patients with type 2 diabetes (T2D) who have established cardiovascular diseases (CVDs) or need to minimize hypoglycemia or promote weight loss [1], it is commonly observed that GLP-1 ra is initiated in the later treatment course of T2D in real-world practice settings as the third-or fourth-line therapy after failure of multiple glucose-lowering agents (GLAs) due to several reasons [2-4]. GLP-1ra is a newer class of GLAs, and thus patients with T2D have been exposed to various GLAs before GLP-1ra became available to them. Also, physicians' or patients' adoption of new drugs may not be as fast as expected due to uncertain long-term real-world drug effectiveness and safety evidence. For instance, a recent study found that less than $10 \%$ of the real-world patients who had comparable CVD risks with the participants in the Liraglutide Effect and Action in Diabetes: Evaluation of Cardiovascular Outcome Results (LEADER) trial were actually prescribed with liraglutide [5]. Moreover, GLP-1 ra is administrated by subcutaneous injection, which likely discourages physicians or patients from the early initiation of GLP-1 ra until a 
therapy with multiple oral GLAs has failed. Also, considering the high acquisition cost of GLP-1 ra, Taiwan's National Health Insurance program implements a restricted reimbursement policy to limit the use of GLP-1ra only for those who have already failed to dual therapy with metformin and sulfonylureas.

Cardiovascular outcomes trials (CVOTs) have found cardiovascular benefits associated with GLP-1 ra [611]. However, there are two important caveats about these findings: 1) study cohorts included in these randomized controlled trials (RCTs) were highly selective, thus reflecting only a subset of the real-world T2D population, and 2) the comparator drug in these RCTs was a placebo instead of an active GLA. In addition, although real-world evidence of the cardiovascular safety of GLP-1 ra has emerged, the incident new-user cohort design was applied in existing studies to only include treatment-naïve users of GLP-1 ra [12-17], which would limit the study generalizability and applicability. Furthermore, only a small portion or even none of Asian populations was included in published RCTs and observational studies. These highlight a research gap in the evaluation of the effects of GLP-1 ra versus other classes of GLAs from the perspective of a more diverse T2D population in the real world, consisting of both patients who newly initiate GLP-1ra in their earlier T2D treatment courses and those who have failed with multiple GLAs and then switched to GLP-1ra.

The present study therefore evaluates the comparative cardiovascular effects of GLP-1 ra in head-to-head comparisons with dipeptidyl peptidase-4 inhibitor (DPP-4i), sulfonylurea (SU), and insulin using the prevalent new-user cohort design [18] to include a broad spectrum of real-world adults with T2D being treated with GLP-1ra to enhance the generalizability of study findings.

\section{Methods}

\section{Data source}

This was a retrospective cohort study utilizing Taiwan's National Health Insurance Research Database (NHIRD) 2003-2015. The NHIRD is derived from the claims data of the National Health Insurance (NHI) program, which covers over $99 \%$ of Taiwan's population (with approximately 23 million people insured) and provides de-identified longitudinal medical and prescription information for each enrolled beneficiary [19].

\section{Cohort identification}

People with newly-diagnosed T2D were identified in 2003-2014 if they had: (1) at least one inpatient record with T2D diagnosis (International Classification of Diseases, Ninth Revision, Clinical Modification [ICD-9-CM]: $250 . X 0$ or 250.X2, where X $=0-9$ ), (2) at least two outpatient records with T2D diagnosis within a given year, or (3) one outpatient record with T2D diagnosis and prescription records of GLAs within a given year. Patients who were aged $<18$ years at T2D diagnosis were excluded. Next, stable users of each study GLA (i.e., GLP-1ra, DPP-4i, SU, or insulin) in 2011-2014 were identified; the period 20112014 was chosen because GLP-1 ra was reimbursed by the NHI program since 2011, and the period ended in 2014 to allow at least one-year follow-up for study subjects. Stable users were defined as patients who 
had at least one stable use set of the study GLA, which was defined as at least three sequential refills of the GLA after its initiation and a prescription gap between any two sequential refills was less than 30 days. A stable user of the study GLA can have multiple stable sets of that drug used chronologically. The study cohort selection flowchart is shown in Fig. 1.

To increase the chances of individual GLP-1ra users being matched with the most comparable users of comparators (i.e., DPP-4i, SU, or insulin) who had the most similar characteristics (i.e., T2D severity, complications, comorbidities, and prior treatments), we allowed stable use sets from a comparator GLA user to be re-used through a three-step matching process (Additional file 1: Figure S1). First, the index date was defined as the initiation date of GLP-1 ra for GLP-1 ra stable users or the first prescription date of stable use set for comparator GLA users in 2011-2014. Based on the availability of GLP-1ra in Taiwan (i.e., 2011), we aligned the cohort entry time for study groups to reduce immortal time bias and minimize confounding effects due to changes in the evolution of GLA treatment and clinical management over time. That is, for each GLP-1ra stable user, we identified stable use sets of a comparator GLA user with the index dates falling within \pm 180 days surrounding the index date of the GLP-1ra user. Second, we adjusted previous utilization patterns/extent of all GLAs, including metformin, SU, meglitinides, thiazolidinediones, acarbose, DPP-4i, and insulin, at one year before GLP-1ra initiation. The utilization of individual GLAs was measured as the total day supply of a drug in the year before the index date. We matched previous utilization patterns/extent of GLAs between GLP-1 ra and comparator GLA users, in which a maximum difference of 90 days supplied (i.e., \pm 45 days) of each class of GLAs between groups was allowed. Lastly, the one-to-one seven-greedy propensity score matching (PSM) was used to adjust for the imbalance of baseline confounder patient characteristics between groups. The propensity score was estimated using a logistic regression model where treatment status (i.e., GLP-1 ra versus comparator GLA) was treated as the dependent variable, and a comprehensive list of clinical characteristics (Table 1) related to either the selection of GLA treatment or study outcomes was identified as independent variables. The second and third steps above ensured that the between-group comparison derived from two groups of patients would have comparable disease severity and previous medication use patterns/extent. To minimize the computational demand for large sample size in the SU group $(21,135,786$ stable use sets of $\mathrm{SU})$ in the matching process, we randomly sampled $30 \%$ of stable use sets of SU for matching with GLP-1ra stable users. 
Table 1

Baseline patient characteristics for different glucose-lowering agent groups after the matching algorithm ${ }^{a}$

\begin{tabular}{|c|c|c|c|c|c|c|}
\hline Characteristics & $\begin{array}{l}\text { GLP- } \\
1 \mathrm{ra}\end{array}$ & $\begin{array}{l}1: 1 \\
\text { matched } \\
\text { DPP-4i }\end{array}$ & $\begin{array}{l}\text { GLP- } \\
\text { 1ra }\end{array}$ & $\begin{array}{l}1: 1 \\
\text { matched } \\
\text { SU }\end{array}$ & $\begin{array}{l}\text { GLP- } \\
\text { 1 ra }\end{array}$ & $\begin{array}{l}\text { 1:1 } \\
\text { matched } \\
\text { insulin }\end{array}$ \\
\hline Number of subjects & 1,893 & 1,893 & 1,829 & 1,829 & 1,367 & 1,367 \\
\hline $\begin{array}{l}\text { Age at index date (years, mean } \pm \\
\text { SD) }\end{array}$ & $\begin{array}{l}49.48 \\
\pm \\
11.61\end{array}$ & $\begin{array}{l}51.78 \pm \\
12.19\end{array}$ & $\begin{array}{l}49.34 \\
\pm \\
11.64\end{array}$ & $\begin{array}{l}51.27 \pm \\
11.82\end{array}$ & $\begin{array}{l}49.38 \\
\pm \\
12.06^{\ddagger}\end{array}$ & $\begin{array}{l}53.07 \pm \\
13.67^{\mathrm{b}}\end{array}$ \\
\hline Male at index date (\%) & 47.97 & 47.17 & 46.04 & 49.7 & 41.7 & 51.43 \\
\hline $\begin{array}{l}\text { Diabetes duration }{ }^{\mathrm{C}} \text { (years, mean } \pm \\
\text { SD) }\end{array}$ & $\begin{array}{l}6.18 \\
\pm 2.74\end{array}$ & $\begin{array}{l}6.46 \pm \\
2.7\end{array}$ & $\begin{array}{l}5.9 \pm \\
2.82\end{array}$ & $\begin{array}{l}6.32 \pm \\
2.74\end{array}$ & $\begin{array}{l}6.37 \pm \\
2.75\end{array}$ & $\begin{array}{l}6.33 \pm \\
2.78\end{array}$ \\
\hline \multicolumn{7}{|l|}{ Comorbidity history (\%) } \\
\hline Hypertension & 62.60 & 62.07 & 61.73 & 62.66 & 59.77 & 67.08 \\
\hline Hyperlipidemia & 70.79 & 71.05 & 70.42 & 70.59 & 69.42 & 70.37 \\
\hline Stroke or transient ischemic attack & 4.86 & 5.23 & 4.37 & 6.07 & 5.27 & 5.05 \\
\hline Heart failure & 2.85 & 3.49 & 2.19 & 3.17 & 3.58 & 3.44 \\
\hline Myocardial infarction & 1.53 & 1.06 & 1.20 & 1.53 & 1.32 & 2.19 \\
\hline Ischemic heart diseases & 12.20 & 11.62 & 12.3 & 13.83 & 13.90 & 14.26 \\
\hline \multicolumn{7}{|l|}{ CIC category (\%) } \\
\hline Cancer & 4.07 & 5.65 & 4.48 & 5.14 & 5.34 & 5.78 \\
\hline Gastrointestinal & 27.63 & 26.62 & 25.48 & 26.46 & 26.63 & 30.43 \\
\hline
\end{tabular}

Abbreviations: CIC, chronic illness with complexity; CVD, cardiovascular disease; DPP-4i, dipeptidyl peptidase-4 inhibitor; GLP-1ra, glucagon-like peptide-1 receptor agonist; MPR, medication possession ratio; RAAS, renin-angiotensin-aldosterone system SD, standard deviation; SU, sulfonylurea;.

${ }^{a}$ All confounders listed above were measured in the year prior to index date, except age, gender, and diabetes duration, which were determined at index date.

${ }^{\mathrm{b}}$ A significant difference between GLP-1ra and insulin users, as indicated by absolute standardized mean difference $>0.2$.

CDiabetes duration was measured as the time from the first date of type 2 diabetes diagnosis to index date.

dMPR was measured as the sum of prescription refill days in the year prior to index date divided by 365. 


\begin{tabular}{|c|c|c|c|c|c|c|}
\hline Characteristics & $\begin{array}{l}\text { GLP- } \\
1 \text { ra }\end{array}$ & $\begin{array}{l}1: 1 \\
\text { matched } \\
\text { DPP-4i }\end{array}$ & $\begin{array}{l}\text { GLP- } \\
1 \text { ra }\end{array}$ & $\begin{array}{l}1: 1 \\
\text { matched } \\
\text { SU }\end{array}$ & $\begin{array}{l}\text { GLP- } \\
1 \text { ra }\end{array}$ & $\begin{array}{l}1: 1 \\
\text { matched } \\
\text { insulin }\end{array}$ \\
\hline Musculoskeletal & 32.70 & 34.28 & 33.13 & 32.31 & 34.67 & 35.70 \\
\hline Pulmonary & 7.82 & 8.45 & 7.27 & 8.37 & 7.39 & 11.49 \\
\hline Substance abuse complexity & 2.54 & 3.06 & 2.41 & 2.62 & 3.00 & 2.49 \\
\hline Mental illness & 8.51 & 8.82 & 8.75 & 10.5 & 10.02 & 10.02 \\
\hline \multicolumn{7}{|l|}{ Diabetes-related complications (\%) } \\
\hline Retinopathy & 17.91 & 17.38 & 16.79 & 18.48 & 21.58 & 19.09 \\
\hline Nephropathy & 27.21 & 27.63 & 24.93 & 27.23 & 27.58 & 28.75 \\
\hline Neuropathy & 14.37 & 15.27 & 13.61 & 15.36 & 16.83 & 17.56 \\
\hline Peripheral vascular diseases & 4.75 & 4.54 & 4.54 & 4.81 & 4.90 & 5.78 \\
\hline Cerebrovascular diseases & 3.96 & 4.38 & 3.39 & 5.03 & 3.95 & 4.39 \\
\hline Cardiovascular diseases & 14.95 & 14.69 & 14.71 & 17.00 & 16.46 & 18.07 \\
\hline Metabolic complications & 0.85 & 2.17 & 1.04 & 1.48 & 2.56 & 2.71 \\
\hline $\begin{array}{l}\text { Number of glucose-lowering agents } \\
\text { prescribed one year before index } \\
\text { date }\end{array}$ & 3.19 & 3.34 & 3.22 & 3.56 & 3.21 & 3.39 \\
\hline \multicolumn{7}{|c|}{ Glucose-lowering agents one year before index date (MPR, mean $\pm S D)^{d}$} \\
\hline Metformin & $\begin{array}{l}0.50 \\
\pm 0.43\end{array}$ & $\begin{array}{l}0.50 \pm \\
0.43\end{array}$ & $\begin{array}{l}0.59 \\
\pm 0.41\end{array}$ & $\begin{array}{l}0.59 \pm \\
0.41\end{array}$ & $\begin{array}{l}0.46 \pm \\
0.43\end{array}$ & $\begin{array}{l}0.46 \pm \\
0.43\end{array}$ \\
\hline
\end{tabular}

\begin{abstract}
Abbreviations: CIC, chronic illness with complexity; CVD, cardiovascular disease; DPP-4i, dipeptidyl peptidase-4 inhibitor; GLP-1 ra, glucagon-like peptide-1 receptor agonist; MPR, medication possession ratio; RAAS, renin-angiotensin-aldosterone system SD, standard deviation; SU, sulfonylurea;.
\end{abstract}

${ }^{a}$ All confounders listed above were measured in the year prior to index date, except age, gender, and diabetes duration, which were determined at index date.

${ }^{\mathrm{b}} \mathrm{A}$ significant difference between GLP-1ra and insulin users, as indicated by absolute standardized mean difference $>0.2$.

'Diabetes duration was measured as the time from the first date of type 2 diabetes diagnosis to index date.

dMPR was measured as the sum of prescription refill days in the year prior to index date divided by 365. 


\begin{tabular}{|c|c|c|c|c|c|c|}
\hline Characteristics & $\begin{array}{l}\text { GLP- } \\
1 \text { ra }\end{array}$ & $\begin{array}{l}1: 1 \\
\text { matched } \\
\text { DPP-4i }\end{array}$ & $\begin{array}{l}\text { GLP- } \\
1 \text { ra }\end{array}$ & $\begin{array}{l}1: 1 \\
\text { matched } \\
\text { SU }\end{array}$ & $\begin{array}{l}\text { GLP- } \\
1 \mathrm{ra}\end{array}$ & $\begin{array}{l}\text { 1:1 } \\
\text { matched } \\
\text { insulin }\end{array}$ \\
\hline Sulfonylurea & $\begin{array}{l}0.43 \\
\pm 0.43\end{array}$ & $\begin{array}{l}0.43 \pm \\
0.43\end{array}$ & $\begin{array}{l}0.66 \\
\pm 0.37\end{array}$ & $\begin{array}{l}0.67 \pm \\
0.37\end{array}$ & $\begin{array}{l}0.32 \pm \\
0.41\end{array}$ & $\begin{array}{l}0.32 \pm \\
0.41\end{array}$ \\
\hline Meglitinide & $\begin{array}{l}0.04 \\
\pm 0.18\end{array}$ & $\begin{array}{l}0.04 \pm \\
0.18\end{array}$ & $\begin{array}{l}0.02 \\
\pm 0.10\end{array}$ & $\begin{array}{l}0.02 \pm \\
0.10\end{array}$ & $\begin{array}{l}0.05 \pm \\
0.19\end{array}$ & $\begin{array}{l}0.05 \pm \\
0.19\end{array}$ \\
\hline Thiazolidinedione & $\begin{array}{l}0.12 \\
\pm 0.28\end{array}$ & $\begin{array}{l}0.12 \pm \\
0.28\end{array}$ & $\begin{array}{l}0.13 \\
\pm 0.30\end{array}$ & $\begin{array}{l}0.14 \pm \\
0.30\end{array}$ & $\begin{array}{l}0.08 \pm \\
0.23\end{array}$ & $\begin{array}{l}0.08 \pm \\
0.23\end{array}$ \\
\hline Acarbose & $\begin{array}{l}0.15 \\
\pm 0.31\end{array}$ & $\begin{array}{l}0.15 \pm \\
0.31\end{array}$ & $\begin{array}{l}0.13 \\
\pm 0.29\end{array}$ & $\begin{array}{l}0.13 \pm \\
0.29\end{array}$ & $\begin{array}{l}0.13 \pm \\
0.28\end{array}$ & $\begin{array}{l}0.13 \pm \\
0.28\end{array}$ \\
\hline DPP-4i & $\begin{array}{l}0.67 \\
\pm 0.34\end{array}$ & $\begin{array}{l}0.67 \pm \\
0.35\end{array}$ & $\begin{array}{l}0.42 \\
\pm 0.42\end{array}$ & $\begin{array}{l}0.42 \pm \\
0.42\end{array}$ & $\begin{array}{l}0.34 \pm \\
0.40\end{array}$ & $\begin{array}{l}0.34 \pm \\
0.40\end{array}$ \\
\hline Insulin & $\begin{array}{l}0.24 \\
\pm 0.38\end{array}$ & $\begin{array}{l}0.24 \pm \\
0.38\end{array}$ & $\begin{array}{l}0.21 \\
\pm 0.36\end{array}$ & $\begin{array}{l}0.21 \pm \\
0.37\end{array}$ & $\begin{array}{l}0.67 \pm \\
0.36\end{array}$ & $\begin{array}{l}0.67 \pm \\
0.36\end{array}$ \\
\hline \multicolumn{7}{|c|}{ CVD-related medication history (\%) } \\
\hline Lipid-modifying agents & 68.94 & 68.57 & 66.21 & 67.2 & 67.15 & 69.35 \\
\hline a-blockers & 4.12 & 4.28 & 3.50 & 2.90 & 3.80 & 4.10 \\
\hline$\beta$-blockers & 31.91 & 30.85 & 31.66 & 31.93 & 33.21 & 35.48 \\
\hline Agents acting on RAAS & 43.69 & 42.37 & 45.05 & 44.18 & 42.28 & 43.75 \\
\hline Diuretics & 18.28 & 18.01 & 20.01 & 20.07 & 21.36 & 18.36 \\
\hline Calcium channel blockers & 32.86 & 31.01 & 32.75 & 33.84 & 32.92 & 35.92 \\
\hline Antiarrhythmics & 1.37 & 1.85 & 1.31 & 1.91 & 1.32 & 2.12 \\
\hline Cardiac glycosides & 0.69 & 1.43 & 0.77 & 0.82 & 0.80 & 1.54 \\
\hline \multicolumn{7}{|c|}{$\begin{array}{l}\text { Abbreviations: CIC, chronic illness with complexity; CVD, cardiovascular disease; DPP-4i, dipeptidyl } \\
\text { peptidase-4 inhibitor; GLP-1 ra, glucagon-like peptide-1 receptor agonist; MPR, medication possession } \\
\text { ratio; RAAS, renin-angiotensin-aldosterone system SD, standard deviation; SU, sulfonylurea;. }\end{array}$} \\
\hline \multicolumn{7}{|c|}{$\begin{array}{l}\text { aAll confounders listed above were measured in the year prior to index date, except age, gender, and } \\
\text { diabetes duration, which were determined at index date. }\end{array}$} \\
\hline \multicolumn{7}{|c|}{$\begin{array}{l}{ }^{b} \text { A significant difference between GLP-1ra and insulin users, as indicated by absolute standardized } \\
\text { mean difference }>0.2 \text {. }\end{array}$} \\
\hline \multicolumn{7}{|c|}{$\begin{array}{l}\text { 'Diabetes duration was measured as the time from the first date of type } 2 \text { diabetes diagnosis to index } \\
\text { date. }\end{array}$} \\
\hline \multicolumn{7}{|c|}{$\begin{array}{l}\text { dMPR was measured as the sum of prescription refill days in the year prior to index date divided by } \\
365 .\end{array}$} \\
\hline
\end{tabular}




\begin{tabular}{|c|c|c|c|c|c|c|}
\hline Characteristics & $\begin{array}{l}\text { GLP- } \\
1 \text { ra }\end{array}$ & $\begin{array}{l}1: 1 \\
\text { matched } \\
\text { DPP-4i }\end{array}$ & $\begin{array}{l}\text { GLP- } \\
1 \text { ra }\end{array}$ & $\begin{array}{l}1: 1 \\
\text { matched } \\
\text { SU }\end{array}$ & $\begin{array}{l}\text { GLP- } \\
1 \text { ra }\end{array}$ & $\begin{array}{l}\text { 1:1 } \\
\text { matched } \\
\text { insulin }\end{array}$ \\
\hline $\begin{array}{l}\text { Vasodilators used in cardiac } \\
\text { diseases }\end{array}$ & 8.19 & 9.67 & 7.93 & 9.51 & 9.73 & 10.31 \\
\hline Anti-platelets & 28.84 & 30.27 & 28.05 & 31.00 & 30.94 & 33.50 \\
\hline Anti-coagulants & 1.16 & 1.69 & 0.87 & 1.09 & 1.61 & 1.76 \\
\hline \multicolumn{7}{|c|}{$\begin{array}{l}\text { Abbreviations: CIC, chronic illness with complexity; CVD, cardiovascular disease; DPP-4i, dipeptidyl } \\
\text { peptidase-4 inhibitor; GLP-1 ra, glucagon-like peptide-1 receptor agonist; MPR, medication possession } \\
\text { ratio; RAAS, renin-angiotensin-aldosterone system SD, standard deviation; SU, sulfonylurea;. }\end{array}$} \\
\hline \multicolumn{7}{|c|}{$\begin{array}{l}\text { a All confounders listed above were measured in the year prior to index date, except age, gender, and } \\
\text { diabetes duration, which were determined at index date. }\end{array}$} \\
\hline \multicolumn{7}{|c|}{$\begin{array}{l}{ }^{\mathrm{b}} \mathrm{A} \text { significant difference between GLP-1ra and insulin users, as indicated by absolute standardized } \\
\text { mean difference }>0.2 \text {. }\end{array}$} \\
\hline \multicolumn{7}{|c|}{$\begin{array}{l}\text { 'Diabetes duration was measured as the time from the first date of type } 2 \text { diabetes diagnosis to index } \\
\text { date. }\end{array}$} \\
\hline \multicolumn{7}{|c|}{$\begin{array}{l}\text { dMPR was measured as the sum of prescription refill days in the year prior to index date divided by } \\
365 .\end{array}$} \\
\hline
\end{tabular}

The exposure to GLAs was measured using the World Health Organization Anatomical Therapeutic Chemical Classification system. The primary outcome was the composite CVDs with fatal/non-fatal events of myocardial infarction (MI), ischemic heart disease, heart failure, ischemic and hemorrhagic stroke, cardiogenic shock, sudden cardiac arrest, arteriosclerotic cardiovascular disease, or arrhythmia. Secondary outcomes included (1) all-cause death, (2) fatal CVDs, and (3) three-point major adverse cardiovascular event (MACE), including non-fatal MI, non-fatal stroke, or fatal CVDs. Using ICD-9-CM codes, cardiovascular outcomes were identified from inpatient and emergency department claims files in the NHIRD. The accuracy of ICD-9-CM coding for study outcomes in the NHIRD has been validated in previous studies [20-24]. The mortality status was ascertained from death cause records from the Ministry of Health and Welfare. Detailed information of the operational definitions is provided in Additional file 2: Table S1. Each patient was followed from the index date until the occurrence of study outcomes, discontinuation of study drugs, death, lost to follow-up from the NHI program, or the end of 2015, whichever came first.

\section{Statistical analysis}

Baseline patient characteristics were measured at one year before or at the index date. Differences in baseline patient characteristics between study groups were compared using the standardized mean 
difference (SMD), where SMD values $>0.2$ were considered to indicate a statistically significant difference between groups $[25,26]$. Cox proportional hazard models were used to estimate the risk of study outcomes between GLP-1 ra and comparator GLAs. Further, subgroup analyses were performed by including interaction terms of study groups and clinical characteristics as covariates in Cox models. A sensitivity analysis was performed where the cutoff point of statistically significant differences in baseline patient characteristics between the drug groups was re-defined as SMD $>0.1$ and thus those variables remaining significantly different after PSM were further adjusted in Cox models. A two-tail pvalue of less than 0.05 was considered statistically significant. All analyses were performed using SAS software version 9.4 .

\section{Results}

There were 3,195 stable users of GLP-1 ra identified in the period 2011-2014. Before the matching, the GLP-1 ra users were generally younger, with a higher proportion being hyperlipidemic and a lower proportion having existing CVDs, compared to the comparator GLA users (Additional file 3: Table S2.). After the matching algorithm was applied, there were a total of $1,893,1,829$, and 1,367 matched pairs of GLP-1 ra users with DPP-4i, SU, and insulin users, respectively. There was no significant difference in baseline patient characteristics between the study groups after the matching, except that the GLP-1ra users were significantly younger than insulin users (Table 1).

Table 2 shows that the event rates of all four study outcomes in GLP-1ra users were lower than those in the three other matched GLA groups. For example, the event rates of the primary CVD composite outcome

for the use of GLP-1ra versus the use of DPP-4i, SU, and insulin were 34.25 vs. $46.20,31.10$ vs. 40.24, and 43.31 vs. 65.88 per 1,000 person-years, respectively. 
Table 2

Event rates of study outcomes associated with the use of GLP-1 ra versus other glucose-lowering agents

\begin{tabular}{|c|c|c|c|c|c|c|}
\hline & $\begin{array}{l}\text { GLP-1 ra } \\
(n= \\
1,893)\end{array}$ & $\begin{array}{l}\text { 1:1 matched } \\
\text { DPP-4i } \\
(n=1,893)\end{array}$ & $\begin{array}{l}\text { GLP-1 ra } \\
(n= \\
1,829)\end{array}$ & $\begin{array}{l}1: 1 \\
\text { matched } \\
\text { SU } \\
(n= \\
1,829)\end{array}$ & $\begin{array}{l}\text { GLP-1ra } \\
(n= \\
1,367)\end{array}$ & $\begin{array}{l}1: 1 \\
\text { matched } \\
\text { insulin } \\
(n= \\
1,367)\end{array}$ \\
\hline \multicolumn{7}{|l|}{ Composite CVD ${ }^{a}$} \\
\hline Number of events & 92 & 141 & 83 & 127 & 78 & 171 \\
\hline $\begin{array}{l}\text { Total person-years in } \\
\text { follow-up }\end{array}$ & $2,686.18$ & $3,051.80$ & $2,669.19$ & $3,155.88$ & $1,800.99$ & $2,595.79$ \\
\hline $\begin{array}{l}\text { Crude rate (per } 1000 \\
\text { person-years) }\end{array}$ & 34.25 & 46.20 & 31.10 & 40.24 & 43.31 & 65.88 \\
\hline \multicolumn{7}{|l|}{ All-cause mortality } \\
\hline Number of events & 1 & 22 & 2 & 6 & 2 & 30 \\
\hline $\begin{array}{l}\text { Total person-years in } \\
\text { follow-up }\end{array}$ & $2,755.76$ & $3,188.36$ & $2,737.90$ & $3,275.08$ & $1,855.12$ & $2,793.32$ \\
\hline $\begin{array}{l}\text { Crude rate (per } 1000 \\
\text { person-years) }\end{array}$ & 0.36 & 6.90 & 0.73 & 1.83 & 1.08 & 10.74 \\
\hline \multicolumn{7}{|l|}{ Fatal CVD } \\
\hline Number of events & 1 & 13 & 2 & 3 & 1 & 9 \\
\hline $\begin{array}{l}\text { Total person-years in } \\
\text { follow-up }\end{array}$ & $2,755.76$ & $3,188.76$ & $2,737.90$ & $3,275.21$ & $1,855.16$ & $2,794.25$ \\
\hline $\begin{array}{l}\text { Crude rate (per } 1000 \\
\text { person-years) }\end{array}$ & 0.36 & 4.08 & 0.73 & 0.92 & 0.54 & 3.22 \\
\hline \multicolumn{7}{|l|}{ MACE $^{b}$} \\
\hline Number of events & 28 & 59 & 29 & 43 & 21 & 68 \\
\hline $\begin{array}{l}\text { Total person-years in } \\
\text { follow-up }\end{array}$ & $2,740.75$ & $3,148.35$ & $2,721.35$ & $3,235.44$ & $1,843.70$ & $2,722.47$ \\
\hline $\begin{array}{l}\text { Crude rate (per } 1000 \\
\text { person-years) }\end{array}$ & 10.22 & 18.74 & 10.66 & 13.29 & 11.39 & 24.98 \\
\hline
\end{tabular}




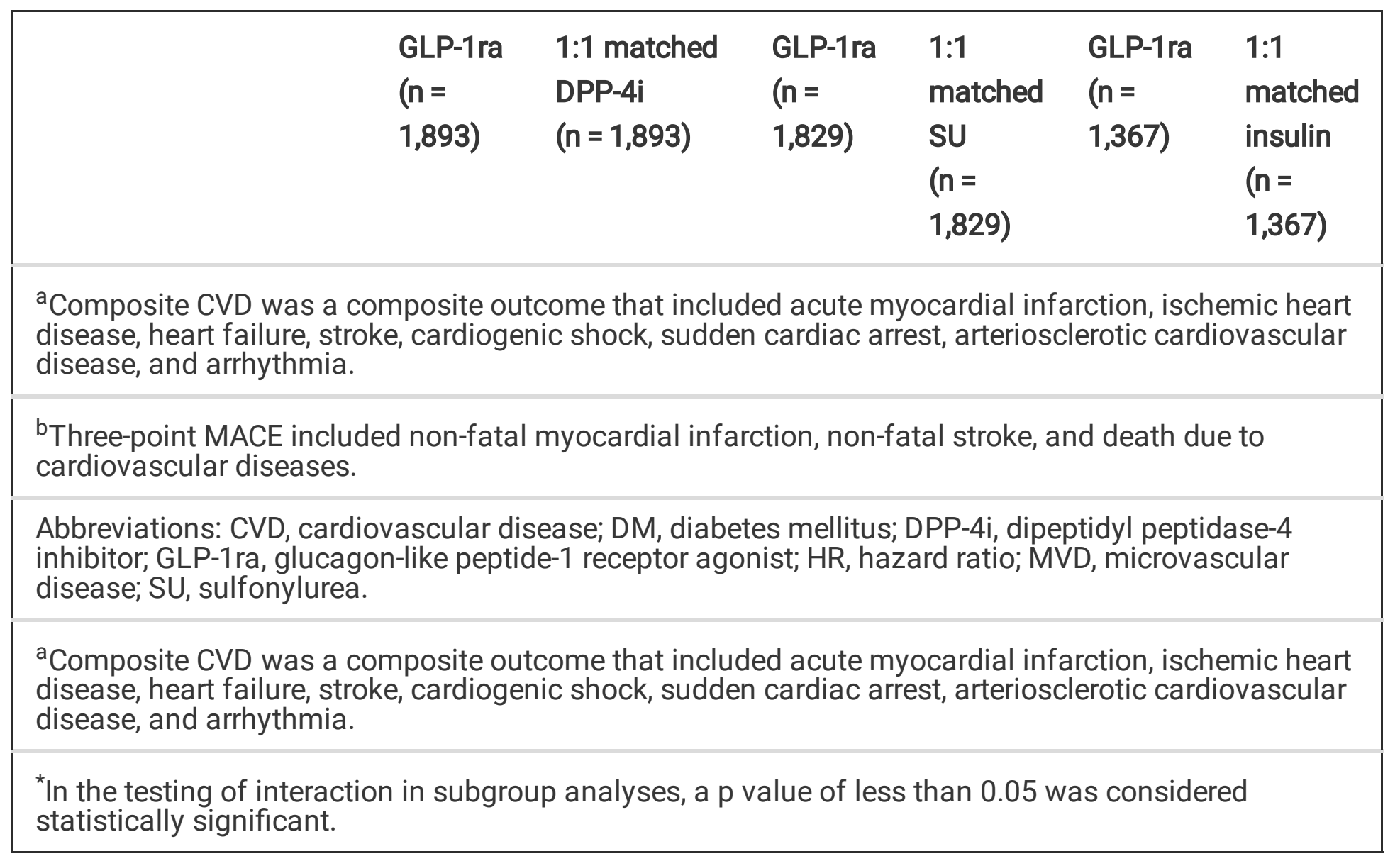

As illustrated in Fig. 2, primary analyses show that the hazard ratios (HRs) and 95\% Cls for the composite CVD were $0.73(0.57-0.96 ; p=.02), 0.76(0.57-1.00 ; p=.05), 0.81(0.62-1.07 ; p=.14)$ for GLP-1 ra use versus DPP-4i, SU, and insulin use, respectively. As shown in Additional file 4: Figure S2, the assessment of three-point MACE reveals that the HRs and $95 \%$ Cls for GLP-1 ra use versus DPP-4i, SU, and insulin use were $0.55(0.35-0.86 ; p=.0087), 0.79(0.49-1.26 ; p=.32), 0.62(0.37-1.02 ; p=.06)$, respectively.

The results of subgroup analyses based on five patient characteristics (i.e., prior history of CVDs, prior history of microvascular diseases, age, gender, and diabetes duration) for the cardiovascular effect of GLP-1 ra versus other GLAs are shown in Fig. 2 and Additional file 4: Figure S2 There was some heterogeneity for the primary outcome; a significant interaction was observed for the absence versus presence of established CVDs at baseline, with a greater benefit in reducing the composite CVD of using GLP-1 ra versus DPP-4i for those without established CVDs at baseline. In contrast, there was a consistent benefit of GLP-1 ra versus other GLAs on three-point MACE across all subgroups. In addition, as shown in original primary and subgroup analyses, sensitivity analyses using a different cutoff point for SMD (i.e., > 0.1 in PSM reveal consistent results that compared to three other GLAs, GLP-1 ra was associated with a lower risk of the composite CVD and three-point MACE (Additional file 5: Table S3. And Additional file 6: Table S4).

\section{Discussion}


This study included a broad representation of the real-world T2D population being treated with GLP-1ra by using the prevalent new-user cohort design to assess its cardiovascular safety compared to three commonly-used classes of GLAs in Taiwan. The majority (87\%) of our study population was those who had failed with more than two types of GLAs and then initiated GLP-1ra. Our results show that GLP-1ra was associated with: 1) a significant cardiovascular benefit in the composite CVD outcome compared to DPP-4i and SU and a non-significantly lower risk of that compared to insulin, and 2) a significant lower risk of three-point MACE compared to DPP-4i and a non-significantly lower risk of that compared to SU and insulin. In addition, prior CVD history was a significant effect-modifier in the association between the use of GLP-1ra versus DPP-4i and the risk of the composite CVD outcome.

Currently, there are no published large, long-term RCTs to evaluate the comparative CVD safety of GLP-1ra in head-to-head comparisons with other active GLAs. The Glycemia Reduction Approaches in Diabetes: A Comparative Effectiveness Study (GRADE) is an ongoing, pragmatic RCT to make head-to-head comparisons of GLP-1 ra, DPP-4i, SU, and basal insulin in metformin-monotherapy patients with relatively recently-diagnosed T2D on glycemia-lowering effectiveness and patient-centered outcomes [27]. However, the GRADE may not be sufficiently powered to evaluate cardiovascular outcomes, and the GRADE participants are initiated with GLP-1ra in their earlier GLA treatment courses and are not representative of real-world patients.

There is the emerging but limited real-world evidence on the comparative cardiovascular safety of GLP1ra [12-17]. Among these studies, while two of them [12,13] included only exenatide in the GLP-1 ra user group in comparison with other GLA user groups, four other real-world observational studies are more relevant to our research. O'Brien et al. used the US nationwide administrative claims data for a retrospective cohort study among insured adults with T2D who newly started second-line GLAs after taking either metformin alone or no prior GLA, and found that the risk of composite CVD events (including ischemic heart disease, congestive heart failure, stroke, or peripheral artery disease, but not cardiovascular death) was significantly lower in GLP-1 ra users compared to DPP-4i users (HR: 0.78, 95\% Cl: 0.63-0.96) [14]. Svanström et al. performed a nationwide register-based cohort study in Denmark and Sweden, which included patients with T2D who newly initiated liraglutide or DPP-4i, and found that the risk of three-point MACE was significantly lower in liraglutide users compared to DPP-4i users (HR: 0.90, 95\% Cl: 0.83-0.98) [15]. Mogensen et al. conducted a nationwide register-based cohort study in Denmark, which included T2D patients without prior MI or stroke that were newly initiated with a combination of metformin with GLP-1 ra or SU, and revealed that the risk of three-point MACE was non-significantly lower in GLP-1 ra users compared to SU users (HR: 0.82, 95\% Cl: 0.55-1.21) [16]. Patorno et al. conducted a cohort study using a US commercial health plan database performed head-to-head comparisons of propensity-score-matched incident new users of GLP-1ra versus DPP-4i, SU, and insulin, and reported nonsignificant differences in the composite CVD events (including acute myocardial infarction, unstable angina, ischemic or hemorrhagic stroke, or coronary revascularization, but not cardiovascular death) between study groups; the HRs $(95 \% \mathrm{Cl})$ were 1.20 (0.76-1.89), $1.05(0.63-1.74)$, and 1.01 (0.73-1.41) for GLP-1ra versus DPP-4i, SU, and insulin, respectively [17]. 
Noticeably, these previous studies used the incident new-user cohort design to only include patients who newly initiated GLP-1ra or the comparator GLAs in the earlier treatment course of T2D, whereas our study utilized the prevalent new-user cohort design to additionally include patients who initiated GLP-1 ra or the comparator GLAs in the later treatment course of T2D. For example, the majority of the previous study cohort $[14,16,17]$ was only on metformin monotherapy before GLP-1ra initiation, whereas most of our study population had already received two or more GLAs before using GLP-1ra. Furthermore, the proportion of patients with comorbidities (e.g., dyslipidemia) and macro- and micro-vascular complications (e.g., CVDs, nephropathy, neuropathy, and retinopathy) in the previous studies $[15,17]$ was lower than that in our study population. Thus, adding to previous studies that focused on the cardiovascular effects of GLP-1ra in the earlier treatment course of T2D, we extend evidence on the cardiovascular safety profile of GLP-1ra to support its rational use in a broader spectrum of real-world T2D patients being treated with GLP-1ra.

Moreover, we observed a significant interaction between the status of prior CVD history and the use of GLP-1ra versus DPP-4i. Compared with DPP-4i, GLP-1 ra significantly lowered the risk of composite CVD events by $49 \%$ in patients without established CVDs, but it had a non-significant risk reduction of $3 \%$ in those with established CVDs. The LEADER trial also reported a significant interaction between prior CVD history and the treatment of liraglutide versus placebo on the risk of three-point MACE, but it showed a greater cardiovascular benefit associated with liraglutide among patients with established CVD compared to those without established CVD before GLP-1 ra initiation [6]. The inconsistent results between our study and the LEADER trial may be explained by: 1) the different compositions of study cohorts between these two studies and 2) the different comparator drugs used in these two studies. For instance, the trial patients tend to be older and have longer diabetes duration compared to our study cohort (i.e., mean age of liraglutide users in the LEADER trial vs. our study: 64.2 vs. 50 years old; mean diabetes duration in the LEADER trial vs. our study: 12.8 vs. 6 years). In addition, the majority (81.3\%) of the LEADER trial patients had established CVDs. This implies that the LEADER trial cohort had severer diabetes and was at higher risk of developing CVDs compared to our study cohort. Therefore, based on current available evidence, one may conclude that GLP-1 ra initiation would yield a cardiovascular benefit for both those with higher CVD risks or established CVDs (from the LEADER trial) and those without established CVDs but already failing with multiple GLAs (from our study).

The present study has several strengths. First, the implementation of the prevalent new-user cohort design to construct the study cohort is essential to ensure the comprehensiveness assessment of the role of GLP-1ra in a diverse real-world population of patients in the earlier or later treatment courses of T2D. It also ensures the completeness of the estimation of the real-world cardiovascular effects of GLP-1 ra and greatly enhances the generalizability of this study to real-world settings. Second, a rigorous three-step matching algorithm was applied in our study cohort selection process to ensure comparability between study groups and to minimize potential time-related problems that are challenging in real-world comparative drug effect studies. Specifically, the index year matching, which aligns cohort entry time between study groups, enhanced comparability between study groups and reduced time-related bias (e.g., different clinical practices or advances in health technology over time). A matching procedure that 
achieves a balance in prior GLA history and disease conditions between study groups allows betweengroup comparisons starting from comparable status of diabetes severity, in which time-lag bias can be reduced $[28,29]$. Third, prior GLA history in this study was measured based on the medication refill data, which could be a surrogate indicator for patients' health behaviors (e.g., drug refill behaviors). For instance, patients who have high persistence or adherence to medication refills may be more likely to engage in healthier behaviors compared to those that do not [30]. Thus, adjustment for the medication refill pattern might allow us to control for potential variations in patients' health behaviors between study groups and thus minimize potential healthy user bias and its effect on study estimates. Lastly, a series of subgroup and sensitivity analyses that considered plausible real-world scenarios of GLP-1ra use ensured the robustness of the study results.

Several limitations should also be acknowledged. First, like other observational studies using administrative claims data, the residual bias attributable to unmeasured confounders (e.g., physicians' preferences, laboratory data) might exist. However, with careful adjustments of disease severity/conditions and prior GLA use, we may reduce the unmeasured confounding that is commonly seen in claims-based studies. Second, medication non-adherence (e.g., short-term or accidental use of drugs of interest) is challenging in real-world observational studies. We applied the stable user definition from our previous studies [31-36] to restrict the analysis to stable GLA users, which might eliminate potential bias introduced from the accidental use or non-adherence of a drug. Third, the generalizability of the study results may be limited to countries with universal health insurance coverage. Lastly, sodiumglucose co-transporter-2 inhibitors were not available in Taiwan's National formulary before May 1, 2016, and thus were not included in analyses.

\section{Conclusions}

Our study findings extend supporting evidence for the cardiovascular safety of GLP-1 ra in head-to-head comparisons with DPP-4i, SU, and insulin to a broad representation of the real-world T2D population using GLP-1ra. The use of GLP-1ra versus DPP-4i may yield a greater cardiovascular benefit in patients without established CVDs compared to those with established CVDs.

\section{Abbreviations}

CVD

cardiovascular disease

CVOT

cardiovascular outcomes trial

DPP-4i

dipeptidyl peptidase-4 inhibitor

GLA

glucose-lowering agent

GLP-1ra 
glucagon-like peptide-1 receptor agonist

GRADE

Glycemia Reduction Approaches in Diabetes:A Comparative Effectiveness Study

ICD-9-CM

International Classification of Diseases, Ninth Revision, Clinical Modification

MACE

major adverse cardiovascular event

$\mathrm{NHI}$

National Health Insurance

NHIRD

National Health Insurance Research Database

RCT

randomized controlled trial

SMD

standardized mean difference

SU

sulfonylurea

T2D

type 2 diabetes

\section{Declarations}

Ethics approval and consent to participate

The study was approved by the Institutional Review Board of National Cheng Kung University Hospital. (A-EX-106-013)

\section{Consent for publication}

Not applicable.

\section{Availability of data and materials}

Data sharing is not applicable to this study as data management and analysis were only allowed to be conducted in Health and Welfare Data Science Center in Taiwan for data privacy and safety concerns.

\section{Competing interests}

No competing interests to be declared.

\section{Funding}


This project was supported by grants from the Ministry of Science and Technology in Taiwan (grant MOST 107-2320-B-006-034) (Huang-Tz Ou) and from the Michigan Center for Diabetes Translational Research (MCDTR) funded by the National Institute of Diabetes and Digestive and Kidney Diseases in the United States (grant P30DK092926) (Shihchen Kuo). The funders had no role in the design, conduct, or reporting of the study.

\section{Author's contributions}

Conception and design: C.T. Yang, H.T. Ou, S. Kuo

Analysis and interpretation of the data: C.T. Yang, C.Y. Yang, H.T. Ou, S. Kuo

Drafting of the article: C.T. Yang, H.T. Ou, S. Kuo

Critical revision of the article for important intellectual content: C.T. Yang, C.Y. Yang, H.T. Ou, S. Kuo

Final approval of the article: C.T. Yang, C.Y. Yang, H.T. Ou, S. Kuo

Provision of study materials or patients: H.T. Ou

Statistical expertise: C.T. Yang, C.Y. Yang, S. Kuo

Administrative, technical, or logistic support: H.T. Ou

Collection and assembly of data: C.T. Yang, C.Y. Yang, H.T.

\section{Acknowledgements}

Not applicable.

\section{References}

1. American Diabetes Association. 9. Pharmacologic approaches to glycemic treatment: Standards of Medical Care in Diabetes-2019. Diabetes Care. 2019; 42(Suppl.1):S90-S102.

2. Otto T, Myland M, Jung H, Lebrec J, Richter $\mathrm{H}$, Norrbacka K. Utilization patterns of glucagon-like peptide-1 receptor agonists in patients with type 2 diabetes mellitus in Germany: a retrospective cohort study. Curr Med Res Opin. 2019;35(5):893-901.

3. Federici MO, McQuillan J, Biricolti G, et al. Utilization patterns of glucagon-like peptide-1 receptor agonists in patients with type 2 diabetes mellitus in Italy: a retrospective cohort study. Diabetes Ther. 2018;9(2):789-801.

4. Persson F, Bodegard J, Lahtela JT, et al. Different patterns of second-line treatment in type 2 diabetes after metformin monotherapy in Denmark, Finland, Norway and Sweden (D360 Nordic): A multinational observational study. Endocrinol Diabetes Metab. 2018;1(4):e00036.

5. Hinton W, Feher M, Munro N, Walker M, de Lusignan S. Real-world prevalence of the inclusion criteria 
for the LEADER trial: data from a national general practice network. Diabetes Obes Metab. 2019;21(7):1661-1667.

6. Marso SP, Daniels GH, Brown-Frandsen K, et al.; LEADER Steering Committee; LEADER Trial Investigators. Liraglutide and cardiovascular outcomes in type 2 diabetes. N Engl J Med.

2016;375(4):311-322.

7. Marso SP, Bain SC, Consoli A, SUSTAIN-6 Investigators, et al. Semaglutide and Cardiovascular Outcomes in Patients with Type 2 Diabetes. N Engl J Med. 2016;375(19):1834-1844.

8. Husain M, Birkenfeld AL, Donsmark M, PIONEER 6 Investigators, et al. Oral Semaglutide and Cardiovascular Outcomes in Patients with Type 2 Diabetes. N Engl J Med. 2019;381(9):841-851.

9. Hernandez AF, Green JB, Janmohamed S, Harmony Outcomes committees and investigators, et al. Albiglutide and cardiovascular outcomes in patients with type 2 diabetes and cardiovascular disease (Harmony Outcomes): a double-blind, randomised placebo-controlled trial. Lancet. 2018; 392(10157):1519-1529.

10. Gerstein HC, Colhoun HM, Dagenais GR, REWIND Investigators, et al. Dulaglutide and cardiovascular outcomes in type 2 diabetes (REWIND): a double-blind, randomised placebo-controlled trial. Lancet. 2019;394(10193):121-130.

11. Cefalu WT, Kaul S, Gerstein HC, et al. Cardiovascular Outcomes Trials in Type 2 Diabetes: Where Do We Go From Here? Reflections From a Diabetes Care Editor's Expert Forum. Diabetes Care. 2018;41(1):1431.

12. Best JH, Hoogwerf BJ, Herman WH, et al. Risk of cardiovascular disease events in patients with type 2 diabetes prescribed the glucagon-like peptide 1 (GLP-1) receptor agonist exenatide twice daily or other glucose-lowering therapies: a retrospective analysis of the LifeLink database. Diabetes Care.

$2011 ; 34(1): 90-95$.

13. Paul SK, Klein K, Maggs D, Best JH. The association of the treatment with glucagon-like peptide-1 receptor agonist exenatide or insulin with cardiovascular outcomes in patients with type 2 diabetes: a retrospective observational study. Cardiovasc Diabetol. 2015;14(1):10.

14. O’Brien MJ, Karam SL, Wallia A, et al. Association of Second-line Antidiabetic Medications With Cardiovascular Events Among Insured Adults With Type 2 Diabetes. JAMA Netw Open. 2018;1(8):e186125.

15. Svanström H, Ueda P, Melbye M, et al. Use of liraglutide and risk of major cardiovascular events: a register-based cohort study in Denmark and Sweden. Lancet Diabetes Endocrinol. 2019;7(2):106-114. 16. Mogensen UM, Andersson C, Fosbøl EL, et al. Cardiovascular safety of combination therapies with incretin-based drugs and metformin compared with a combination of metformin and sulphonylurea in type 2 diabetes mellitus-a retrospective nationwide study. Diabetes Obes Metab. 2014;16(10):1001-1008. 17. Patorno E, Everett BM, Goldfine AB, et al. Comparative cardiovascular safety of glucagon-like peptide1 receptor agonists versus other antidiabetic drugs in routine care: a cohort study. Diabetes Obes Metab. 2016;18(8):755-765.

18. Suissa S, Moodie EE, Dell'Aniello S. Prevalent new-user cohort designs for comparative drug effect studies by time-conditional propensity scores. Pharmacoepidemiology and drug safety. 2017;26(4):459468. 
19. Cheng TM. Taiwan's new national health insurance program: genesis and experience so far. Health Aff (Millwood). 2003;22(3):61-76.

20. Hsieh CY, Su CC, Shao SC, et al. Taiwan's National Health Insurance Research Database: past and future. Clin Epidemiol. 2019;11:349-358.

21. Cheng CL, Kao YH, Lin SJ, Lee CH, Lai ML. Validation of the National Health Insurance Research Database with ischemic stroke cases in Taiwan. Pharmacoepidemiol Drug Saf. 2011;20(3):236-242. 22. Cheng CL, Lee CH, Chen PS, Li YH, Lin SJ, Yang YH. Validation of acute myocardial infarction cases in the National Health Insurance Research database in Taiwan. J Epidemiol. 2014;24(6):500-507.

23. Wu CS, Lai MS, Gau SS, Wang SC, Tsai HJ. Concordance between patient self-reports and claims data on clinical diagnoses, medication use, and health system utilization in Taiwan. PLoS One. 2014;9(12): e112257.

24. Sung SF, Hsieh CY, Lin HJ, Chen YW, Yang YH, Li CY. Validation of algorithms to identify stroke risk factors in patients with acute ischemic stroke, transient ischemic attack, or intracerebral hemorrhage in an administrative claims database. Int J Cardiol. 2016;215:277-282.

25. Peter C. Austin. Using the Standardized Difference to Compare the Prevalence of a Binary Variable Between Two Groups in Observational Research. Commun Stat Simul Comput. 2009;38(6):1228-1234. 26. Yang D, Dalton JE. A unified approach to measuring the effect size between two groups using SAS®. SAS Global Forum 2012: Statistics and Data Analysis, Paper 335-2012.

27. Nathan DM, Buse JB, Kahn SE, GRADE Study Research Group, et al. Rationale and design of the glycemia reduction approaches in diabetes: a comparative effectiveness study (GRADE). Diabetes Care. 2013;36(8):2254-2261.

28. Suissa S. Lower Risk of Death With SGLT2 Inhibitors in Observational Studies: Real or Bias? Diabetes Care. 2018;41(1):6-10.

29. Suissa S, Azoulay L. Metformin and the risk of cancer: time-related biases in observational studies. Diabetes Care. 2012;35(12):2665-2673.

30. Matthews KA, Kuller LH, Wing RR, Meilahn EN, Plantinga P. Prior to use of estrogen replacement therapy, are users healthier than nonusers? Am J Epidemiol. 1996;143(10):971-978.

31. Ou HT, Chang KC, Li CY, Wu JS. Risks of cardiovascular diseases associated with dipeptidyl peptidase-4 inhibitors and other antidiabetic drugs in patients with type 2 diabetes: a nation-wide longitudinal study. Cardiovasc Diabetol. 2016;15:41.

32. Ou HT, Chang KC, Li CY, Wu JS. Comparative cardiovascular risks of dipeptidyl peptidase 4 inhibitors with other second- and third-line antidiabetic drugs in patients with type 2 diabetes. $\mathrm{Br} \mathrm{J}$ Clin Pharmacol. 2017;83(7):1556-1570.

33. Hou WH, Chang KC, Li CY, Ou HT. Dipeptidyl peptidase-4 inhibitor use is not associated with elevated risk of severe joint pain in patients with type 2 diabetes: a population-based cohort study. Pain. 2016;157(9):1954-1959.

34. Lu CH, Yang CY, Li CY, Hsieh CY, Ou HT. Lower risk of dementia with pioglitazone, compared with other second-line treatments, in metformin-based dual therapy: a population-based longitudinal study. Diabetologia. 2018;61(3):562-573.

35. Hou WH, Chang KC, Li CY, Ou HT. Dipeptidyl peptidase-4 inhibitor use is associated with decreased 
risk of fracture in patients with type 2 diabetes: a population-based cohort study. $\mathrm{Br} \mathrm{J}$ Clin Pharmacol. 2018;84(9):2029-2039.

36. Ou HT, Chen YT, Liu YM, Wu JS. Comparative cost-effectiveness of metformin-based dual therapies associated with risk of cardiovascular diseases among Chinese patients with type 2 diabetes: Evidence from a population-based national cohort in Taiwan. Diabetes Res Clin Pract. 2016;116:14-25.

\section{Figures}


Newly diagnosed with type 2 diabetes in period 2003-2014

$$
(\mathrm{N}=2,338,292)
$$

Patients who were prescribed with glucose-lowering agents (GLAs) at least

$$
\text { once in period 2011-2014 }
$$

$\checkmark$ Glucagon-like peptide-1 receptor agonist (GLP-1 ra): 4,125

$\checkmark$ Dipeptidyl peptidase-4 inhibitor (DPP-4i): 415,763

$\checkmark$ Sulfonylurea (SU): 929,240

$\checkmark$ Insulin: 281,210

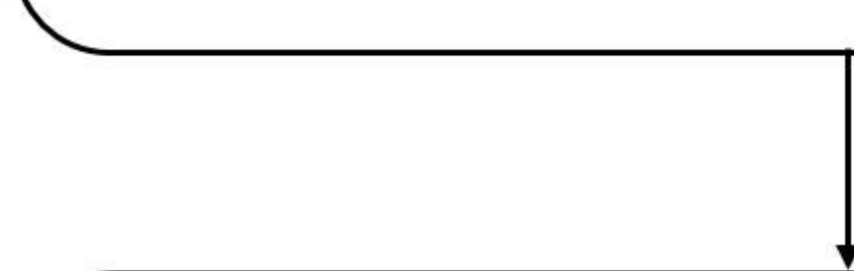

Patients who were stably prescribed with GLAs in period 2011-2014

$\checkmark$ GLP-1ra:3,195

$\checkmark$ DPP-4i:388,011

$\checkmark \quad$ SU: 773,026

$\checkmark$ Insulin: 155,473

Figure 1

Flowchart of study population selection and identification 


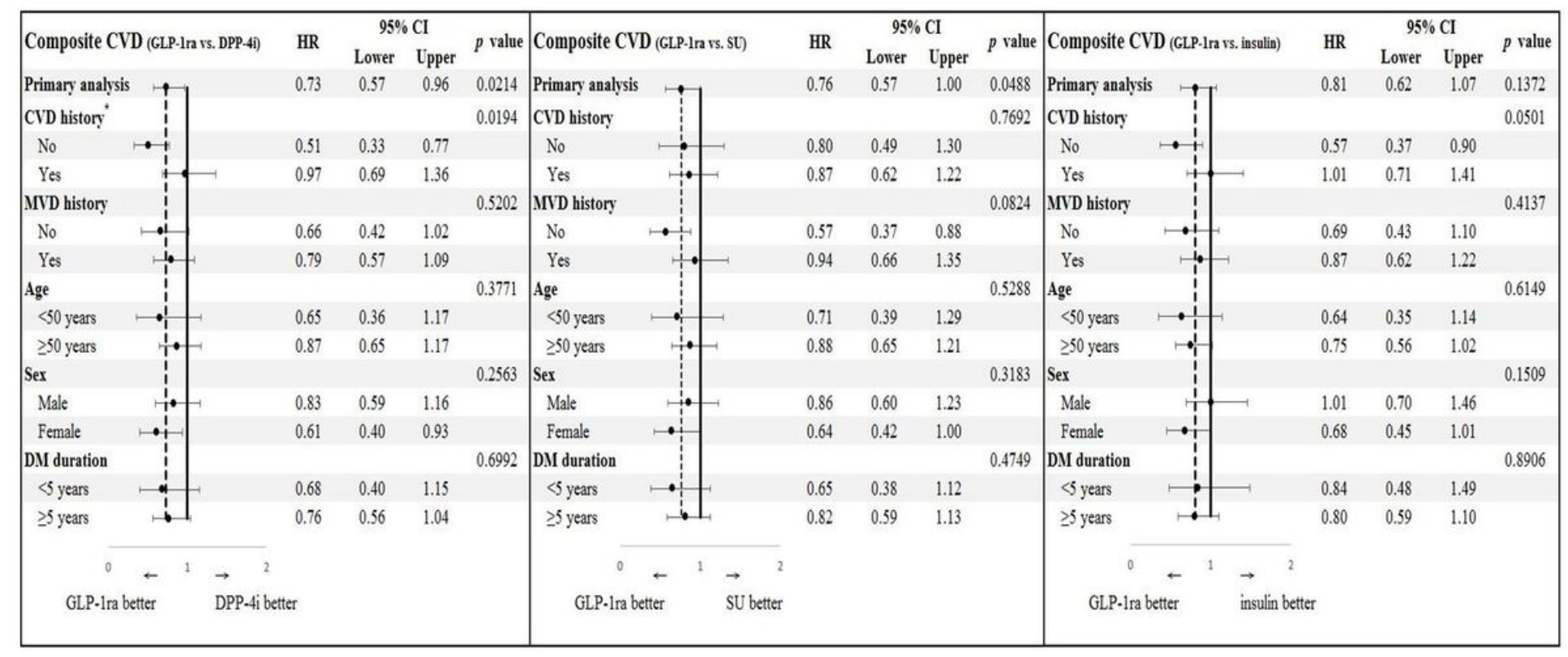

\section{Figure 2}

Primary and subgroup analyses for composite CVD associated with GLP-1 ra versus other glucoselowering agentsa Abbreviations: CVD, cardiovascular disease; DM, diabetes mellitus; DPP-4i, dipeptidyl peptidase-4 inhibitor; GLP-1 ra, glucagon-like peptide-1 receptor agonist; HR, hazard ratio; MVD, microvascular disease; SU, sulfonylurea. aComposite CVD was a composite outcome that included acute myocardial infarction, ischemic heart disease, heart failure, stroke, cardiogenic shock, sudden cardiac arrest, arteriosclerotic cardiovascular disease, and arrhythmia.

\section{Supplementary Files}

This is a list of supplementary files associated with this preprint. Click to download.

- Additionalfile6TableS4.docx

- Additionalfile4FigureS2.20200224.docx

- Additionalfile2TableS1.docx

- Additionalfile3Tables2.docx

- Additionalfile5Tables3.docx

- Additionalfile1FigureS1.20200220.docx 